\title{
Cone Avoidance of Some Turing Degrees
}

\author{
Patrizio Cintioli ${ }^{1}$ \\ ${ }^{1}$ Dipartimento di Matematica e Informatica, Università di Camerino, Camerino, Italy \\ Correspondence: Patrizio Cintioli, Dipartimento di Matematica e Informatica, Via Madonna delle Carceri, 9, 62032 \\ Camerino(MC), Italy. E-mail: patrizio.cintioli@unicam.it
}

Received: May 25, 2017 Accepted: June 19, 2017 Online Published: July 13, 2017

doi:10.5539/jmr.v9n4p69 URL: https://doi.org/10.5539/jmr.v9n4p69

\begin{abstract}
We study the cone avoidance and the upper cone avoidance of two substructures of $m$-introimmune Turing degrees. We show that the substructure of the $m$-introimmune Turing degrees satisfies the cone avoidance property, and that the substructure of the computably enumerable $m$-introimmune Turing degrees satisfies the upper cone avoidance property.
\end{abstract}

Keywords: Turing degrees, cone avoidance, upper cone avoidance.

\section{Introduction}

The study of sets of natural numbers with no subsets of higher Turing degrees started with Soare (Soare 1969) and continued with Jockusch (Jockusch 1973) and Simpson (Simpson 1978). From their works we know that such sets exist and that they cannot be arithmetic. On the other hand, for stronger reducibilities $\leq_{r}$ there are arithmetic sets with no subsets of higher $\leq_{r}$-degrees (Cintioli and Silvestri 2003), (Ambos-Spies 2003). We recall that a reducibility is stronger if it is strictly contained in the Turing reducibility.

Given one of the common reducibility $\leq_{r}$ studied in computability theory, a possible way to define the property of having no subsets of higher $\leq_{r}$-degrees is that of being $r$-introimmune, a property introduced in (Cintioli and Silvestri 2003): an infinite set $A$ of natural numbers is $r$-introimmune if for every $B \subseteq A$ with $|A-B|=\infty$ is $A \not_{r} B$.

We know that for some stronger reducibilities $\leq_{r}$ there are arithmetic $r$-introimmune sets. In particular, there are $m$ introimmune sets in the class $\Pi_{1}^{0}$ (Cintioli 2005), where $m$ stands for the many-one reducibility $\leq_{m}$. This suggests to study which properties satisfies the substructure of the computably enumerable (c.e.) $m$-introimmune Turing degrees. Let $\mathbf{I}_{m}=\{\mathbf{a}: \mathbf{a}$ is a c.e. Turing degree containing a $m$-introimmune set $\}$, and let $\mathcal{I}_{m}=\left(\mathbf{I}_{m}, \leq\right)$ be the substructure of the c.e. $m$-introimmune Turing degrees. For example, we know of this substructure that it has no minimum and it has maximum (Cintioli 2005). Moreover, a portion of $\mathcal{I}_{m}$ is an upper semilattice (Cintioli 2014). In section 3 we continue the study of the substructure $I_{m}$ by considering the upper cone avoidance property. The upper cone avoidance property is one of the main properties studied in the structure of the c.e. Turing degrees, and we prove that $I_{m}$ satisfies this property.

In section 4 we consider the substructure $\mathcal{J}_{m}=\left(\mathbf{J}_{m}, \leq\right)$ of the $m$-introimmune Turing degrees, that is the structure where $\mathbf{J}_{m}=\{\mathbf{a}: \mathbf{a}$ is a Turing degree containing a $m$-introimmune set $\}$. We know that $\mathcal{J}_{m}$ has minimal elements and that it does not have maximum. We consider here the cone avoidance property, and we prove that $\mathcal{J}_{m}$ satisfies this property.

\section{Notations and Preliminaries}

For the notations we refer to the Soare's book (Soare, 1987). Letter $\mathbb{N}$ denotes the set of natural numbers. Given two sets $A, B \subseteq \mathbb{N}$, the complement of $A$ is denoted by $\bar{A}$, while $A-B$ denotes the difference of $A$ and $B$. For every set $A \subseteq \mathbb{N}$ and for every natural number $n, A\lceil n=A \cap\{0,1, \ldots, n\}$. We identify the characteristic function of a set $A$ with $A$ itself, that is $A(x)=1$ if $x \in A$ and $A(x)=0$ if $x \notin A$, for every $x \in \mathbb{N}$. We use the standard enumeration of all the Turing functionals $\Phi_{0}, \Phi_{1}, \ldots$, where for every $e \in \mathbb{N}$ and for every $X \subseteq \mathbb{N} \Phi_{e}^{X}$ is the partial function computated by the $e$-th oracle Turing machine with the aid of the oracle $X$. We write $\Phi_{e}^{A}(x) \downarrow$ if the $e$-th oracle Turing machine with oracle $A$ halts on input $x$. For every natural numbers $e, s, x$ and for every oracle $X$ we define $\Phi_{e, s}^{X}(x):=\Phi_{e}^{X}(x)$ if there exists $t \leq s$ such that the $e$-th oracle Turing machine on input $x$ with oracle $X$ halts in exactly $t$ steps; in this case we say that $\Phi_{e, s}^{X}(x)$ is defined and we write $\Phi_{e, s}^{X}(x) \downarrow$; we say that $\Phi_{e, s}^{X}(x)$ is undefined otherwise. We fix also an acceptable enumeration $f_{0}, f_{1}, \ldots$ of all the unary Turing computable functions. $W_{0}, W_{1}, \ldots$ is the corresponding enumeration of all the computably enumerable (c.e.) sets. For every $e, s, x \in \mathbb{N}$ we define $f_{e, s}(x):=f_{e}(x)$ if there exists $t \leq s$ such that the $e$-th Turing machine on input $x$ halts in exactly $t$ steps; in this case we say that $f_{e, s}(x)$ is defined and we write $f_{e, s}(x) \downarrow$; we say that $f_{e, s}(x)$ is undefined otherwise. For every $e, s \in \mathbb{N}, W_{e, s}$ is the finite approximation of $W_{e}$ given by performing $s$ steps in the enumeration of $W_{e}$. Given two sets $A, B \subseteq \mathbb{N}$ : 
- $A$ is many-one reducible to $B$, in short $A \leq_{m} B$, if there exists a total computable function $f: \mathbb{N} \rightarrow \mathbb{N}$ such that for every $x \in \mathbb{N}, x \in A$ if and only if $f(x) \in B$.

- $A$ is Turing reducible to $B$, in short $A \leq_{T} B$, if there exists $e \in \mathbb{N}$ such that $\Phi_{e}^{B}=A$.

The Turing degree of a set $A \subseteq \mathbb{N}$ is the equivalence class $\left\{B \subseteq \mathbb{N}: A \equiv_{T} B\right\}$, where $A \equiv_{T} B$ if and only if $A \leq_{T} B$ and $B \leq_{T} A$. The Turing reducibility $\leq_{T}$ induces the partial ordering $\leq$ on the Turing degrees in the obvious way. Turing degrees, or simply degrees, are denoted with the lower case letters $\mathbf{a}, \mathbf{b}, \ldots$ Two degrees $\mathbf{a}, \mathbf{b}$ are incomparable if $\mathbf{a} \not \mathbf{b}$ and $\mathbf{b} \not \mathbf{a}$, and in this case we write $\mathbf{a} \mid \mathbf{b}$. $\mathbf{0}$ is the Turing degree of the computable sets. The jump of degree $\mathbf{a}$ is $\mathbf{a}^{\prime}$. In particular, $\mathbf{0}^{\prime}$ is the Turing degree of the set $\left\{x \in \mathbb{N}: \Phi_{x}^{\emptyset}(x) \downarrow\right\}$. Given a Turing degree $\mathbf{a}$,

(i) the upper cone of $\mathbf{a}$ is the set $\{\mathbf{b}: \mathbf{a} \leq \mathbf{b}\}$, and

(ii) the cone of $\mathbf{a}$ is the set $\{\mathbf{b}: \mathbf{a} \leq \mathbf{b}\} \cup\{\mathbf{c}: \mathbf{c} \leq \mathbf{a}\}$.

\section{Upper Cone Avoidance}

In this section we consider the substructure $\mathcal{I}_{m}=\left(\mathbf{I}_{m}, \leq\right)$. Of this structure we know that it has no minimum and it has $\mathbf{0}^{\prime}$ as maximum (Cintioli 2005). It is not a lattice because there is a minimal pair in $\mathcal{I}_{m}$ (Cintioli 2011), and we do not know if it is an upper semilattice. Continuing with the research of the properties of $\mathcal{I}_{m}$, we show that it satisfies the upper cone avoidance property, that is, for every degree $\mathbf{c} \in \mathbf{I}_{m}$ there exists $\mathbf{a} \in \mathbf{I}_{m}$ such that $\mathbf{c} \not \mathbf{a}$. The upper cone avoidance property of $\mathcal{I}_{m}$ is an immediate corollary of Theorem 3.2.

Theorem 3.2 For every noncomputable c.e. degree $\mathbf{c}$ there exists a c.e. degree $\mathbf{a} \in \mathbf{I}_{m}$ such that $\mathbf{c} \nless \mathbf{a}$.

Proof. Let $\mathbf{c}$ be a noncomputable c.e. degree and let $C \in \mathbf{c}$ be a c.e. set. Let $\left\{C_{s}\right\}_{s \geq 0}$ be a uniformly recursive sequence of finite sets such that $C=\bigcup_{s \geq 0} C_{s}$, with $C_{s} \subseteq C_{s+1}$ for every $s \geq 0$.

\subsection{Strategy}

We construct by a finite-injury priority argument a c.e. set $A=\bigcup_{s \geq 0} A_{s}$, where $A_{s}$ is the finite set constructed by the end of stage $s$, with $A_{0}=\emptyset$ and $A_{s} \subseteq A_{s+1}$ for every $s \geq 0$. For every $e \in \mathbb{N}$ the set $A$ will satisfy the following requirements:

- $P_{4 e}:\left|W_{e}\right|=\infty \Rightarrow\left[W_{e} \cap A \neq \emptyset\right]$ (immunity of $\bar{A}$ )

- $N_{4 e+1}:(\exists x \geq e)[x \in \bar{A}]$ (infinity of $\bar{A}$ )

- $N_{4 e+2}:(\forall B \subseteq \bar{A})\left[|\bar{A}-B|=\infty \Rightarrow \bar{A}\right.$ is not $m$-reducible to $B$ by $\left.f_{e}\right](m$-introimmunity of $\bar{A})$

- $N_{4 e+3}: \Phi_{e}^{A} \neq C$ (upper cone avoidance).

In our construction we combine the strategy of to make $C \bigsqcup_{T} A$ with the strategy of to make the degree of $A$ in $\mathbf{I}_{m}$. At each stage $s$ of the construction we try to fulfil one requirement, if possible, among those not yet fulfilled. Requirements $\left\{P_{4 e}\right\}_{e \geq 0}$ are positive, because to fulfil them we enumerate elements in $A$. Requirements $\left\{N_{4 e+1}, N_{4 e+3}\right\}_{e \geq 0}$ are negative, because to fulfil them we try to keep elements out of $A$. Requirements $\left\{N_{4 e+2}\right\}_{e \geq 0}$ are positive and negative, because to fulfil them we enumerate elements in $A$ and we try to keep elements out of $A$. From now on letter $\mathcal{R}$ will denote any requirement. At every stage there could be requirements requiring attention. A requirement $\mathcal{R}_{m}$ has higher priority than $\mathcal{R}_{n}$ if $m<n$. We call a requirement $\mathcal{R}_{m}$ active at the stage $s$ if $m$ is the minimum index such that $\mathcal{R}_{m}$ requires attention at stage $s$.

\subsection{Injured Requirements}

At every stage $s$ we try to satisfy a requirement $\mathcal{R}_{m}$. If $m \neq 4 e$ for every $e \in \mathbb{N}$, then we try to satisfy $\mathcal{R}_{m}$ by restraining some element to be enumerated in $A$. To this end, we use a restraint function $r: \mathbb{N}^{2} \rightarrow \mathbb{N}$. A requirement $\mathcal{R}_{m}$ is injured at stage $s$ if an element $y \leq r(m, s)$ is enumerated in $A$. All the positive requirements $\left\{P_{4 e}\right\}_{e \geq 0}$ cannot be injured, so the restraint function is useless for them and we define $r(4 e, s)=-1$ for every $e, s \in \mathbb{N}$.

\subsection{Actions to Fulfil Requirements}

We describe informally the actions to fulfil requirements. To make $C \bigsqcup_{T} A$ we try to satisfy $N_{4 e+3}: \Phi_{e}^{A} \neq C$ for every $e \in \mathbb{N}$. We employ the Sacks' agreement method. This method is based on the definition at each stage $s$ and for every $e \leq s$ of the length agreement function $l(e, s)$ :

$$
l(e, s):=\max \left\{z:(\forall y<z)\left[C_{s}(y)=\Phi_{e, s}^{A_{s}}(y) \downarrow\right]\right\} .
$$


The objective is to ensure that $\lim _{s \rightarrow \infty} l(e, s)=l_{e}$ is finite for every $e \in \mathbb{N}$, which implies $\Phi_{e}^{A}\left(l_{e}\right) \neq C\left(l_{e}\right)$. We need the use function $u\left(A_{s} ; e, x, s\right)$ defined in the following way: $u\left(A_{s} ; e, x, s\right)$ is $1+$ the maximum element asked to $A_{s}$ in the computation of $\Phi_{e, s}^{A_{s}}(x)$ if $\Phi_{e, s}^{A_{s}}(x) \downarrow$, and $u\left(A_{s} ; e, x, s\right)$ is 0 if $\Phi_{e, s}^{A_{s}}(x)$ is undefined. To preserve the computation of $\Phi_{e, s}^{A_{s}}(y)$ for all $y \leq l(e, s)$ we define at each stage $s$ the restraint function

$$
r(4 e+3, s):=\max \left\{u\left(A_{s} ; e, y, s\right): y \leq l(e, s)\right\} .
$$

The finite-injury method will ensure that from an opportune stage $s_{0}$ onwards requirement $N_{4 e+3}$ will not be injured anymore, so

$$
\lim _{t \rightarrow \infty} l(e, t)=l\left(e, s_{0}\right)=l_{e}
$$

This implies that for every $t \geq s_{0}$

$$
\Phi_{e, t}^{A_{t}}(l(e, t))=\Phi_{e, s_{0}}^{A_{s_{0}}}\left(l\left(e, s_{0}\right)\right)=\Phi_{e, s_{0}}^{A_{s_{0}}}\left(l_{e}\right)=\Phi_{e}^{A}\left(l_{e}\right)
$$

and

$$
\Phi_{e}^{A}\left(l_{e}\right) \neq C\left(l_{e}\right) .
$$

To make the degree of $A$ in $\mathbf{I}_{m}$ we try to satisfy every requirement $N_{4 e+2}, e \geq 0$. To satisfy all such requirements we use the strategy of to make $\bar{A}$ immune because of the following Proposition 3.3 proved in (Cintioli, 2011).

Proposition 3.3 (Cintioli 2011) Let $B$ be an immune set, and let $Y \subseteq B$ with $|B-Y|=\infty$ such that $B \leq_{m} Y$ via the computable function $f$. Then the set $\{f(x): f(x) \neq x \wedge x \in B\}$ is infinite.

We will make $\bar{A}$ immune by satisfying all the requirements $\left\{P_{4 e}\right\}_{e \geq 0}$. Thanks to the Proposition 3.3 we try to satisfy each requirement $N_{4 e+2}$ by waiting for a stage $s+1$ such that for some $x \notin A_{s}$ is $f_{e, s}(x) \downarrow \neq x$ and $f_{e, s}(x)$ is not restrained by requirements of higher priority than $N_{4 e+2}$, that is $f_{e, s}(x)>\max _{i<4 e+2}\{r(i, s)\}$. If such a stage is found, we enumerate $f_{e, s}(x)$ in $A$ and keep out $x$ of $A$ by setting $r(4 e+2, s)=x$. In this way $N_{4 e+2}$ is satisfied at stage $s+1$; if $N_{4 e+2}$ is not injured after stage $s+1$ then $f_{e}$ is not a $m$-reduction of $\bar{A}$ to any of its co-infinite subset.

\subsection{Requirements Requiring Attention}

We now define formally when a requirement requires attention. For every $e, s \geq 0$, we say that

- requirement $P_{4 e}$ requires attention at stage $s+1 \geq 4 e$ if

$$
W_{e, s} \cap A_{s}=\emptyset \text { and there is } x \in W_{e, s} \text { with } x>\max _{i<4 e}\{r(i, s)\} .
$$

- Requirement $N_{4 e+1}$ requires attention at stage $s+1 \geq 4 e+1$ if $r(4 e+1, s)=-1$.

- Requirement $N_{4 e+2}$ requires attention at stage $s+1 \geq 4 e+2$ via $x \leq s+1$ if $r(4 e+2, s)=-1$ and

$$
x \in \overline{A_{s}} \wedge f_{e, s}(x) \downarrow \neq x \wedge f_{e, s}(x)>\max _{i<4 e+2}\{r(i, s)\} .
$$

\subsection{Construction of $A$}

Stage $s=0$. Set $A_{0}:=\emptyset$ and $r(m, 0)=-1$ for every $m \geq 0$.

Stage $s+1$. If there are no requirements requiring attention, then do nothing, that this define $A_{s+1}=A_{s}$ and $r(m, s+1):=$ $r(m, s)$ for every $m \neq 4 e+3$, for every $e \geq 0$. Otherwise, let $\mathcal{R}_{n}$ be the active requirement. We distinguish three cases on $\mathcal{R}_{n}$.

- $\mathcal{R}_{n}=P_{4 e}$. Let $x$ be the minimum for which $P_{4 e}$ requires attention. Define $A_{s+1}=A_{s} \cup\{x\}$.

- $\mathcal{R}_{n}=N_{4 e+1}$. Let $x$ be the minimum for which $N_{4 e+1}$ requires attention. Define $r(4 e+1, s+1)=x$ and $A_{s+1}=A_{s}$.

- $\mathcal{R}_{n}=N_{4 e+2}$. Let $x$ be the minimum for which $N_{4 e+2}$ requires attention. Define $A_{s+1}=A_{s} \cup\left\{f_{e, s}(x)\right\}$ and $r(4 e+2, s+1):=x$.

For all $n \neq 4 e+3$ for every $e \geq 0$, if $N_{n}$ is injured then define $r(n, s+1)=-1$, otherwise define $r(n, s+1)=r(n, s)$. 


\section{End construction of $A$}

To prove that set $A$ has the desired properties it suffices to prove that all the requirements are eventually satisfied. We prove first that all the requirements $\left\{P_{4 e}, N_{4 e+1}, N_{4 e+2}\right\}_{e \geq 0}$ require attention at most finitely often. Then we show by the Sacks' agreement method that every negative requirement $N_{4 e+3}$ is fulfilled.

Lemma 3.4 For every e $\in \mathbb{N}$, requirements $P_{4 e}, N_{4 e+1}$ and $N_{4 e+2}$ require attention at most finitely often.

Proof. The proof is by complete induction on $n, n \neq 4 e+3$, for every $e \geq 0$. Given $n$, let $s_{0}$ be the minimum stage such that no requirement $\mathcal{R}_{m}$ with $m<n$ requires attention after stage $s_{0}$. We distinguish three cases on $n$.

- $n=4 e$. Let us suppose that $P_{4 e}$ requires attention at stage $s+1 \geq s_{0}$. By hypothesis $P_{4 e}$ is active at stage $s+1$, so an element $x \in W_{e, s}$ is enumerated in $A_{s+1}$. This means that $A_{t} \cap W_{e, t} \neq \emptyset$ for every $t \geq s+1$, therefore condition (1) will be false at every stage $t \geq s+1$ and $P_{4 e}$ will not require attention anymore.

$-n=4 e+1$. If for every $s \geq s_{0} r(4 e+1, s)>-1$, then $N_{4 e+1}$ does not require attention. Otherwise, let $s^{\prime}+1 \geq s_{0}$ be a stage such that $r\left(4 e+1, s^{\prime}\right)=-1$. By hypothesis $N_{4 e+1}$ is active at this stage and $r\left(4 e+1, s^{\prime}+1\right)=x$ for the minimum $x \geq e$ and $x \notin A_{s}$. After stage $s^{\prime}+1$ no requirement of higher priority of $N_{4 e+1}$ requires attention, so $r(4 e+1, t)=r\left(4 e+1, s^{\prime}+1\right)=x>-1$ for every $t \geq s^{\prime}+1$ and $N_{4 e+1}$ will not receive attention anymore.

- $n=4 e+2$. Let us suppose that $N_{4 e+2}$ requires attention at stage $s+1 \geq s_{0}$. By hypothesis $N_{4 e+2}$ is active at stage $s+1$, so an element $y$ is enumerated in $A_{s+1}$ and $r(4 e+2, s+1)=y$. By hypothesis at every stage $t>s+1$ no requirement $\mathcal{R}_{m}$ with $m<4 e+2$ will require attention, whence no element $\leq r(4 e+2, s+1)=r(4 e+2, t)$ will be enumerated in $A_{t}$. This means that for every $t>s+1$ it holds that $r(4 e+2, t)>-1$, that is $N_{4 e+2}$ will not require attention anymore.

\section{End proof of Lemma 3.4}

Lemma 3.5 For every $e \geq 0$, requirement $N_{4 e+3}$ is met.

Proof. This follows from the length agreement method of G. Sacks. Given $e$, let us suppose for the sake of contradiction that $N_{4 e+3}$ is not met. This means that $\Phi_{e}^{A}=C$, so

$$
\lim _{s \rightarrow \infty} l(e, s)=\infty .
$$

Let $s_{0}$ be a stage such that no requirement $\mathcal{R}_{n}$ with $n<4 e+3$ require attention after $s_{0}$. The following is an informal procedure to decide $C$. Given any number $y$, decide " $y \in C$ " by searching for a stage $s \geq s_{0}$ with $y<l(e, s)$, which exists by (2). Then, decide " $y \in C$ " by computing $\Phi_{e, s}^{A_{s}}(y)$.

In the following claim we prove that the procedure is correct.

Claim 3.6 $C(y)=\Phi_{e, s}^{A_{s}}(y)$.

Proof. At stage $s$ is $r(4 e+3, s)=\max \left\{u\left(A_{s} ; e, x, s\right): x \leq l(e, s)\right\}=r_{e}$. Since no requirement $\mathcal{R}_{m}$ with $m<4 e+3$ is active after stage $s$, it holds that for every $t \geq s$

$$
A_{t} \uparrow r_{e}=A \uparrow r_{e}
$$

so

$$
\Phi_{e, t}^{A_{t} \backslash r_{e}}(y)=\Phi_{e, t}^{A \nmid r_{e}}(y)
$$

However, every computation of $\Phi_{e, t}^{A \mid r_{e}}(y)$ use only elements less than $r_{e}$, so for every $t \geq s$

$$
\Phi_{e, t}^{A\left\lceil r_{e}\right.}(y)=\Phi_{e, t}^{A}(y) .
$$

This implies that for every $t \geq s$

$$
\Phi_{e, t}^{A}(y)=\Phi_{e}^{A}(y)=C(y)
$$

End proof of the claim.

Thus the set $C$ is computable, contrary to the assumption that $\mathbf{c}$ was noncomputable.

\section{End proof of Lemma 3.5 .}

It remains to prove that all the requirements $\left\{P_{4 e}, N_{4 e+1}, N_{4 e+2}\right\}_{e \geq 0}$ are met. We observe first that $\lim _{s \rightarrow \infty} r(4 e+3, s)$ exists and is finite. 
Lemma 3.7 For every $e \in \mathbb{N}, \lim _{s \rightarrow \infty} r(4 e+3, s)$ exists and is finite.

Proof. Fix $e \in \mathbb{N}$. The proof is part of the Sacks' agreement method, and it follows from the fact that $N_{4 e+3}$ is satisfied and that all the requirement $\left\{P_{4 m}, N_{4 m+1}, N_{4 m+2}\right\}_{m \geq 0}$ require attention at most finitely often, that is $N_{4 e+3}$ is not injured after an opportune stage. The interested reader can see also either (Soare 1987, page 123, cf. Lemma 2) or (Odifreddi 1999, page 464 et seq.)

Let $l_{e}$ be the minimum number for which $\Phi_{e}\left(l_{e}\right) \neq C\left(l_{e}\right)$, which exists because $N_{4 e+3}$ is met. Let $s_{0}$ be such that no requirement $\mathcal{R}_{m}$ with $m<4 e+3$ requires attention after $s_{0}$ and such that for every $s \geq s_{0}$,

$$
C_{s}(y)=C(y)
$$

for every $y \leq l_{e}$, and

$$
\Phi_{e, s}^{A_{s}}(y)=\Phi_{e}^{A}(y)
$$

for every $y<l_{e}$. If for every $s \geq s_{0} \Phi_{e, s}^{A_{s}}\left(l_{e}\right)$ is undefined, then

$$
\lim _{s \rightarrow \infty} r(4 e+3, s)=r\left(4 e+3, s_{0}\right) .
$$

Otherwise, if $s_{1}$ is the first stage after $s_{0}$ such that $\Phi_{e, s_{1}}^{A_{s_{1}}}\left(l_{e}\right)$ is defined, then for every $t \geq s_{1}$

$$
\left(\forall y<l_{e}\right)\left[\Phi_{e, t}^{A_{t}}(y)=\Phi_{e}^{A}(y)\right]
$$

because $N_{4 e+3}$ is not injured after $s_{1} \geq s_{0}$. Moreover, by hypothesis $\Phi_{e}^{A}\left(l_{e}\right) \neq C\left(l_{e}\right)$, so $\lim _{s \rightarrow \infty} l(e, s)=l\left(e, s_{0}\right)=l_{e}$ and by the definition of $r(4 e+3, s)$ is $\lim _{s \rightarrow \infty} r(4 e+3, s)=r\left(4 e+3, s_{1}\right)$.

\section{End proof of Lemma 3.7}

Lemma 3.8 For every $e \in \mathbb{N}, P_{4 e}, N_{4 e+1}$ and $N_{4 e+2}$ are met.

Proof. By Lemma 3.4 and Lemma 3.7 it follows that $\lim _{s \rightarrow \infty} r(n, s)$ exists and is finite for every $n \in \mathbb{N}$. To prove the lemma, fix $\mathcal{R}_{n}$ and let $s_{0}$ be the minimum stage after which no requirement $\mathcal{R}_{m}$ with $m<n$ requires attention. Distinguish three cases on $n$.

- $n=4 e$. If $W_{e}$ is finite then $P_{4 e}$ is met, so let us suppose that $W_{e}$ is infinite. If at stage $s_{0}$ is $W_{e, s_{0}} \cap A_{s_{0}} \neq \emptyset$, then $P_{4 e}$ is met. Otherwise, let $s+1>s_{0}$ be the minimum stage such there is $x \in W_{e, s}$ with $W_{e, s} \cap A_{s}=\emptyset$ and $x>\max \{r(n, s): n<4 e\}$. At stage $s+1$ requirement $P_{4 e}$ requires attention and by hypothesis on $s_{0}$ is active. Therefore $x$ is enumerated in $A_{s+1}$ and for every $t \geq s+1$ is $W_{e, t} \cap A_{t} \neq \emptyset$, that is $W_{e} \cap A \neq \emptyset$ and $P_{4 e}$ is met.

- $n=4 e+1$. If $r\left(4 e+1, s_{0}\right)=x \geq e$, then by hypothesis on $s_{0}$ no requirement will enumerate $x$ in $A$, so $x \in \bar{A}$ and $N_{4 e+1}$ is met. If $r\left(4 e+1, s_{0}\right)=-1$, let $s+1$ be the minimum stage with $s+1 \geq \max \left\{s_{0}, 4 e+1\right\}$ and let $x \geq e$ be the minimum element not in $A_{s}$. $N_{4 e+1}$ requires attention and by hypothesis on $s_{0}$ is active at stage $s+1$, so $r(4 e+1, s+1)=x$. After stage $s+1 \geq s_{0}$ requirement $N_{4 e+1}$ is not injured, hence $x \in \overline{A_{t}}$ for every $t \geq s+1$, that is $x \in \bar{A}$ and $N_{4 e+1}$ is met.

- $n=4 e+2$. If the domain of $f_{e}$ is different from $\mathbb{N}$ then $N_{4 e+2}$ is met, so let us suppose that domain of $f_{e}$ is $\mathbb{N}$. For the sake of contradiction let us suppose that $N_{4 e+2}$ is not met, and let $X \subseteq \bar{A}$ such that $\bar{A} \leq_{m} X$ via $f_{e}$, with $|\bar{A}-X|=\infty$. Since for every $e \geq 0$ requirement $P_{4 e}$ is met it follows that $\bar{A}$ is immune. By Proposition 3.3 the set $\left\{f_{e}(x): f_{e}(x) \neq x \wedge x \in \bar{A}\right\}$ is infinite. So, there are a stage $s+1 \geq s_{0}$ and $x \in \mathbb{N}$ such that:

(i) $x \notin A_{s}$,

(ii) $f_{e, s}(x) \downarrow \neq x$ and $f_{e, s}(x)>\max \{r(n, s): n<4 e+2\}$.

Requirement $N_{4 e+2}$ requires attention at stage $s+1$ and by hypothesis on $s_{0}$ is active. This means that $f_{e, s}(x)$ is enumerated in $A_{s+1}$ and $r(4 e+2, s+1)=x$. After stage $s_{0}$ no requirement of higher priority than $N_{4 e+2}$ requires attention, so $N_{4 e+2}$ is never injured, in particular $x \in \overline{A_{t}}$ for every $t \geq s+1 \geq s_{0}$, that is $x \notin A$. This is a contradiction, because $x \in \bar{A}$ and $f_{e}(x) \notin \bar{A}$, which implies $f_{e}(x) \notin X \subseteq \bar{A}$, contrary to the assumption that $f_{e}$ was a $m$-reduction of $\bar{A}$ to $X$.

End proof of Lemma 3.8

This also concludes the proof of the theorem.

Corollary 3.9 In the substructure of the c.e. m-introimmune Turing degrees holds the upper cone avoidance property. 


\section{Cone Avoidance}

In this section we consider the substructure $\mathcal{J}_{m}=\left(\mathbf{J}_{m}, \leq\right)$ of the $m$-introimmune degrees. Of this substructure we know that it has minimal elements and that it does not have maximum. These properties follow from results concerning cohesive degrees. First of all, in (Cintioli 2005) it has been proved that every cohesive set is $m$-introimmune. Then, by (Jockusch 1973) we know that if $\mathbf{b}$ is a cohesive degree and $\mathbf{b} \leq \mathbf{a}$ then $\mathbf{a}$ is a cohesive degree, from which we derive that $\mathcal{J}_{m}$ does not have maximum. Moreover, it follows from (Jockusch 1973, Corollary 2) and (Cooper 1973, Theorem 1) that there are cohesive minimal degrees, so there are minimal elements in $\mathcal{J}_{m}$. We consider the cone avoidance property and we show that $\mathcal{J}_{m}$ satisfies this property, that is for every $\mathbf{b} \in \mathbf{J}_{m}$ there exists $\mathbf{a} \in \mathbf{J}_{m}$ such that $\mathbf{b} \not \mathbf{a}$ and $\mathbf{a} \not \mathbf{b}$. Actually, the degree $\mathbf{a}$ that avoids the cone of degree $\mathbf{b}$ is bi- $m$-introimmune, that is $\mathbf{a}$ contains a set $A$ such that both $A$ and $\bar{A}$ are $m$-introimmune.

For convenience we will use the notion of string to denote finite subsets of $\mathbb{N}$. Formally, a string is a function $\alpha$ : $\{0,1, \ldots, n\} \rightarrow\{0,1\}$, where $n \in \mathbb{N}$. For every $n \in \mathbb{N}$ the length of a string $\alpha:\{0,1, \ldots, n\} \rightarrow\{0,1\}$, in short $|\alpha|$, is $n+1$. Given two strings $\alpha$ and $\beta$, we write:

$$
\begin{aligned}
& -\alpha \subseteq \beta \text { if }|\alpha| \leq|\beta| \text { and } \alpha(m)=\beta(m) \text { for every } m<|\alpha|, \\
& \text { - } \alpha \subset \beta \text { if } \alpha \subseteq \beta \text { and } \alpha \neq \beta \text {. }
\end{aligned}
$$

We represent any string $\alpha:\{0,1, \ldots, n\} \rightarrow\{0,1\}$ with the binary word $\alpha(0) \alpha(1) \cdots \alpha(n)$. If $\alpha:\{0,1, \ldots, n\} \rightarrow\{0,1\}$ and $\beta:\{0,1, \ldots, m\} \rightarrow\{0,1\}$ are two strings, then $\alpha \beta$ denotes the concatenation of $\alpha$ and $\beta$, that is the string of length $|\alpha|+|\beta|$ whose representation is

$$
\alpha(0) \alpha(1) \cdots \alpha(n) \beta(0) \beta(1) \cdots \beta(m) .
$$

Given $b \in\{0,1\}$ and any positive integer $n$, we write $b^{n}$ to represent the string $\alpha$ of length $n$ such that $\alpha(i)=b$ for every $0 \leq i<n$. We will denote strings with letters $\sigma, \tau$. From now on we identify any string $\sigma$ with the set $\{n \in \mathbb{N}: n<$ $|\sigma| \wedge \sigma(n)=1\}$. Furthermore, for every $e, x, t \in \mathbb{N}$ and for every string $\sigma, \Phi_{e, t}^{\sigma}(x) \downarrow$ implies that all the queries made to the oracle $\sigma$ are less than $|\sigma|$.

Theorem 4.1 For every degree $\mathbf{b}>\mathbf{0}$ there exists a bi-m-introimmune degree $\mathbf{a}<\mathbf{b}^{\prime}$ such that $\mathbf{a} \mid \mathbf{b}$.

In order to prove the theorem we introduce some few concepts and a result concerning them.

First of all, we say that a function $f: \mathbb{N} \rightarrow \mathbb{N}$ is injective almost everywhere if the set $\left\{(x, y) \in \mathbb{N}^{2}: x \neq y \wedge f(x)=f(y)\right\}$ is finite.

Definition 4.2 A function $g: \mathbb{N} \rightarrow \mathbb{N}$ is dominating if for every computable total function $f: \mathbb{N} \rightarrow \mathbb{N}$ there exists $m \in \mathbb{N}$ such that

$$
(\forall n>m)[g(n)>f(n)] .
$$

Definition 4.3 (Kämper 1990) Given any set $D \subseteq \mathbb{N}$ and given any strictly increasing function $g: \mathbb{N} \rightarrow \mathbb{N}$, we say that $D$ has $g$-gaps if there are infinitely many $m \in \mathbb{N}$ such that $D \cap\{x: m \leq x<g(m)\}=\emptyset$.

Finally, we define the concept of strongly bi-m-immunity.

Definition 4.4 (Balcázar and Schöning 1985) $A$ set $X \subseteq \mathbb{N}$ is strongly bi- $m$-immune if and only if every $m$-reduction of $X$ to any $Y \subseteq \mathbb{N}$ is injective almost everywhere.

We observe that a set is strongly bi- $m$-immune if and only if its complement is strongly bi- $m$-immune. It is known that a strongly bi- $m$-immune set with $g$-gaps for a dominating function $g$ is $m$-introimmune.

Lemma 4.5 (Cintioli 2005) Let $X$ be a strongly bi- $m$-immune set, and let $g: \mathbb{N} \rightarrow \mathbb{N}$ be a strictly increasing dominating function. If $X$ has $g$-gaps, then $X$ is $m$-introimmune.

Now, we can proceed with the proof of Theorem 4.1.

Proof. Let $B \in \mathbf{b}$, and let $h: \mathbb{N} \rightarrow \mathbb{N}$ be any strictly increasing dominating $\emptyset^{\prime}$-computable function. We construct by the finite-extension method a set $A \leq_{T} B^{\prime}$ which is strongly-bi- $m$-immune, Turing incomparable with $B$, and such that both $A$ and $\bar{A}$ have $h$-gaps. So, by Lemma $4.5 A$ is bi- $m$-introimmune. The construction is by stages. At every stage $s$ we will define a finite string $\sigma_{s}$. The final set $A$ will be $\bigcup_{s \geq 0} \sigma_{s}$, with $\sigma_{0}=\emptyset$ and $\sigma_{s} \subset \sigma_{s+1}$, for every $s \in \mathbb{N}$. The set $A$ will satisfy for every $e \in \mathbb{N}$ the following requirements.

- $G_{e}$ : there are $m_{1}, m_{2} \geq e$ such that $A \cap\left\{x: m_{1} \leq x<h\left(m_{1}\right)\right\}=\emptyset$ and $\bar{A} \cap\left\{x: m_{2} \leq x<h\left(m_{2}\right)\right\}=\emptyset$.

- $H_{e}$ : if $f_{e}$ is not injective almost everywhere, then $f_{e}$ does not $m$-reduce $A$ to any $X$. 


$$
\begin{aligned}
& -R_{e}: \Phi_{e}^{B} \neq A \\
& -S_{e}: \Phi_{e}^{A} \neq B .
\end{aligned}
$$

All the requirements $\left\{G_{e}\right\}_{e \geq 0}$ guarantee that both $A$ and $\bar{A}$ have $h$-gaps and all the requirements $\left\{H_{e}\right\}_{e \geq 0}$ guarantee that $A$ is strongly-bi- $m$-immune. All the requirement $R_{e}$ and $S_{e}$ guarantee that the two sets $A$ and $B$ are Turing incomparable. The construction of $A$ is the following.

4.1 Construction of $A$ (with the aid of oracle $B^{\prime}=\left\{x \in \mathbb{N}: \Phi_{x}^{B}(x) \downarrow\right\}$ ).

Stage 0 . Let $\sigma_{0}$ be the empty string.

Stage $s+1$. Let $\sigma_{s}$ be the string defined at stage $s$ and let $n=\left|\sigma_{s}\right|$. Distinguish four cases on $s+1$.

- Case $s+1=4 e$. In this case we insert a $h$-gap in both $A$ and $\bar{A}$. Set $\sigma_{s+1}=\sigma_{s} 0^{h(n)-n} 1^{h(h(n))-h(n)}$.

- Case $s+1=4 e+1$. In this case we try to satisfy $H_{e}$. Check if

$$
(\exists \sigma)(\exists i)(\exists j)(\exists t)\left[\sigma \supset \sigma_{s} \wedge \sigma(i) \neq \sigma(j) \wedge f_{e, t}(i)=f_{e, t}(j)\right]
$$

If (7) is false then set $\sigma_{s+1}=\sigma_{s} 0$. Otherwise, enumerate the set $\left\{(\sigma, i, j, t): \sigma \supset \sigma_{s}\right.$ and $i, j<|\sigma|$ and $\left.t \in \mathbb{N}\right\}$ until a quadruple $(\sigma, i, j, t)$ satisfying the matrix of the formula (7) is found. Set $\sigma_{s+1}=\sigma$.

- Case $s+1=4 e+2$. In this case we try to satisfy $R_{e}$. Check if $\Phi_{e}^{B}(n) \downarrow$. If not, then set $\sigma_{s+1}=\sigma_{s} 0$. Otherwise, set $\sigma_{s+1}=\sigma_{s} 1$ if $\Phi_{e}^{B}(n)=0$, and $\sigma_{s+1}=\sigma_{s} 0$ if $\Phi_{e}^{B}(n) \neq 0$.

- Case $s+1=4 e+3$. In this case we try to satisfy $S_{e}$. Check if

$$
(\exists \sigma)(\exists \tau)(\exists x)(\exists t)\left[\sigma, \tau \supset \sigma_{s} \wedge \Phi_{e, t}^{\sigma}(x) \downarrow \neq \Phi_{e, t}^{\tau}(x) \downarrow\right] .
$$

If (8) does not hold, then set $\sigma_{s+1}=\sigma_{s} 0$. Otherwise, enumerate the set $\left\{(\sigma, \tau, x, t): \sigma, \tau \supset \sigma_{s}\right.$ and $\left.x, t \in \mathbb{N}\right\}$ until a quadruple $(\sigma, \tau, x, t)$ satisfying the matrix of the formula (8) is found. If $B(x) \neq \Phi_{e, t}^{\sigma}(x)$ then set $\sigma_{s+1}=\sigma$, otherwise set $\sigma_{s+1}=\tau$.

\section{End construction of $A$}

We observe first that for every $s \in \mathbb{N}\left|\sigma_{s+1}\right| \geq\left|\sigma_{s}\right|+1$, which implies that $\left|\sigma_{s}\right| \geq s$. In the next claim we prove that $A$ is $B^{\prime}$-computable.

Claim 4.6 $A \leq_{T} B^{\prime}$.

Proof. It is enough to show that with oracle $B^{\prime}$ it is possible to define $\sigma_{s}$, for every $s \geq 0$. The proof is by induction on $s$. Fix stage $s+1$, and let us suppose that $\sigma_{s}$ can be constructed with oracle $B^{\prime}$. At stage $s+1$ we have to consider four cases.

- Case $s+1=4 e$. In this case we have to compute $h\left(\left|\sigma_{s}\right|\right)$. But by hypothesis $h$ is $\emptyset^{\prime}$-computable, hence $B^{\prime}$-computable.

- Case $s+1=4 e+1$. In this case oracle $\emptyset^{\prime}$ suffices to check test (7), so oracle $B^{\prime}$ suffices.

- Case $s+1=4 e+2$. In this case oracle $B^{\prime}$ suffices to both check whether or no $\Phi_{e}^{B}(n) \downarrow$ and to compute $\Phi_{e}^{B}(n)$.

- Case $s+1=4 e+3$. In this case oracle $\emptyset^{\prime}$ suffices to check test (8), thus $B^{\prime}$ suffices. Furthermore, oracle $B^{\prime}$ suffices to check whether or no $B(x) \neq \Phi_{e, t}^{\sigma}(x)$.

\section{End proof of the Claim 4.6}

Now, it remains to show that all the requirements are met.

Lemma 4.7 For every $e \in \mathbb{N}$ requirements $G_{e}, H_{e}, R_{e}$ and $S_{e}$ are met.

Proof. Fix $e$. For $G_{e}$, let $s+1=4 e$. At this stage we set $\sigma_{s+1}=\sigma_{s} 0^{h(n)-n} 1^{h(h(n))-h(n)}$ with $n=\left|\sigma_{s}\right| \geq s \geq e$. Therefore

$$
\sigma_{s+1}(x)=0 \text { for every }\left|\sigma_{s}\right| \leq x<h\left(\left|\sigma_{s}\right|\right)
$$

and

$$
\sigma_{s+1}(x)=1 \text { for every } h\left(\left|\sigma_{s}\right|\right) \leq x<h\left(h\left(\left|\sigma_{s}\right|\right)\right) \text {. }
$$


From (9) it follows that

$$
A \cap\left\{x:\left|\sigma_{s}\right| \leq x<h\left(\left|\sigma_{s}\right|\right)\right\}=\emptyset
$$

with $\left|\sigma_{s}\right| \geq e$, while from (10) it follows that

$$
\bar{A} \cap\left\{x: h\left(\left|\sigma_{s}\right|\right) \leq x<h\left(h\left(\left|\sigma_{s}\right|\right)\right)\right\}=\emptyset
$$

with $h\left(\left|\sigma_{s}\right|\right) \geq e$. Whence $G_{e}$ is met.

For $H_{e}$, let us suppose that $f_{e}$ is total and not 1-1 almost everywhere, that is the set

$$
I=\left\{(x, y): x \neq y \wedge f_{e}(x)=f_{e}(y)\right\}
$$

is infinite, and let $X$ be any set. This means that there are infinitely many strings $\sigma \supset \sigma_{s}$ and infinitely many $(i, j) \in I$ with $\sigma(i) \neq \sigma(j)$. So at stage $s+1=4 e+1$ test (7) is true. Let $(\sigma, i, j, t)$ be the first quadruple appearing in the subsequent enumeration. Then we set $\sigma_{s+1}=\sigma$. Without loss of generality let us suppose that $\sigma_{s+1}(i)=0$ and $\sigma_{s+1}(j)=1$, that is $i \notin A$ and $j \in A$. Then,

$$
\begin{aligned}
& \text { - if } f_{e}(i)=f_{e}(j) \in X \text {, then } i \notin A \text { and } f_{e}(i) \in X \text {; } \\
& \text { - if } f_{e}(i)=f_{e}(j) \notin X \text {, then } j \in A \text { and } f_{e}(j) \notin X \text {. }
\end{aligned}
$$

Thus $f_{e}$ does not $m$-reduce $A$ to $X$.

For $R_{e}$, let $n=\left|\sigma_{s}\right|$ at stage $s+1=4 e+2$ and let us suppose that $\Phi_{e}^{B}$ is total, which implies that $\Phi_{e}^{B}(n) \downarrow$. Then $\sigma_{s+1}(n) \neq \Phi_{e}^{B}(n)$, that is $n \in A \Leftrightarrow \Phi_{e}^{B}(n)=0$, therefore $\Phi_{e}^{B} \neq A$ and $R_{e}$ is met.

For $S_{e}$, the splitting method ensures that it is met. In fact, for the sake of contradiction let us suppose that $S_{e}$ is not met, which means that

$$
\Phi_{e}^{A}=B
$$

Then we claim that $B$ is computable, contrary to the assumption $\mathbf{b}>\mathbf{0}$. Let $s+1=4 e+3$ and let $\sigma_{s}$ be the string constructed up to the end of stage $s$. By (11), for every $n \in \mathbb{N}$ there are $\sigma \subset A$ and $t \in \mathbb{N}$ such that $\Phi_{e, t}^{\sigma}(n)=B(n)$. The following is an informal algorithm to decide $B$. Given $n \in \mathbb{N}$, enumerate the set $\left\{(\sigma, t): \sigma \supseteq \sigma_{s} \wedge t \in \mathbb{N}\right\}$ and stop when for some $(\sigma, t)$ is $\Phi_{e, t}^{\sigma}(n) \downarrow$. Then

$$
\Phi_{e, t}^{\sigma}(n)=B(n),
$$

because if not then there would be $\tau \subset A, \tau \supseteq \sigma_{s}$, such that $B(n)=\Phi_{e}^{\tau}(n) \neq \Phi_{e}^{\sigma}(n)$. This means that the matrix of the formula of test (8) is satisfied for $\sigma, \tau, n, t$. Since at stage $s+1=4 e+3$ we choose $\sigma_{s+1}$ making $\Phi_{e}^{\sigma_{s+1}}(n) \neq B(n)$, and since $\sigma_{s+1} \subset A$, it follows that $\Phi_{e}^{A}(n) \neq B(n)$, contrary to (11).

This concludes the proof of Lemma 4.7.

Hence $A ⿱_{T} B$ and $B ⿱_{T} A$, with $A, B \leq_{T} B^{\prime}$ and $A$ bi- $m$-introimmune, so the statement of the theorem follows.

Corollary 4.8 In the substructure of the m-introimmune Turing degree holds the cone avoidance property.

\section{References}

Ambos-Spies, K (2003). Problems which cannot be reduced to any proper subproblems. In: Proc. 28th International Symposium MFCS 2003, Lectures Notes in Computer Science (B. Rovan and P. Vojtáš, eds.)(2747), 162-168 (Springer, Berlin, 2003)

Balcázar, J. L. \& Schöning, U. (1985). Bi-immune sets for complexity classes. Mathematical Systems Theory. An International Journal on Mathematical Computing Theory, 18, 1-10.

Cooper, B. (1973). Minimal degrees and the jump operator. The Journal of Symbolic Logic, 38(2), 249-271.

Cintioli, P. (2005). Sets without subsets of higher many-one degrees. Notre Dame J. Form. Log., 46(2), 207-216. http://dx.doi.org/10.1305/ndjfl/1117755150

Cintioli, P. (2011). Low sets without subsets of higher many one degrees. Math. Log. Quart., 57(5), 517-523. http://dx.doi.org/10.1002/malq.200920043

Cintioli, P., \& Silvestri R. (2003). Polynomial time introreducibility. Theory Comput. Syst., 36(1), 1-15. 
http://dx.doi.org/10.1007/s00224-002-1040-z

Jockusch, Jr., C. G. (1973). Upward closure and cohesive degrees. Isr. J. Math., 15, 332-335.

http://dx.doi.org/10.1007/BF02787575

Kämper, J. (1990). A result relating disjunctive self-reducibility to P-immuity. Information Processing Letters, 33(5), 239-242.

Odifreddi, P. (1999). Classical Recursion Theory II, in: Studies in Logic and the Foundations of Mathematics Vol. 143, (North-Holland, Amsterdam,1999).

Simpson, S. G. (1978). Sets which do not have subsets of every higher degree. J. Symb. Log., 43(1), 135-138, (1978). http://dx.doi.org/10.2307/2271956

Soare, R. I. (1987). Recursively enumerable sets and degrees, Perspectives in Mathematical Logic (Springer-Verlag, 1987).

\section{Copyrights}

Copyright for this article is retained by the author(s), with first publication rights granted to the journal.

This is an open-access article distributed under the terms and conditions of the Creative Commons Attribution license (http://creativecommons.org/licenses/by/4.0/). 